\title{
Osteoprotegerin and osteopontin serum values in postmenopausal advanced breast cancer patients treated with anastrozole
}

\author{
A Martinetti ${ }^{1}, E$ Bajetta ${ }^{2}$, L Ferrari ${ }^{1}, N$ Zilembo $^{2}$, E Seregni ${ }^{1}$,

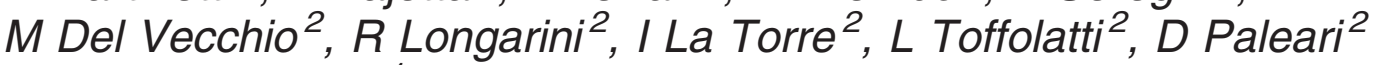 \\ and $\mathrm{E}$ Bombardieri ${ }^{1}$
}

${ }^{1}$ Nuclear Medicine Unit and ${ }^{2}$ Medical Oncology Unit B, Istituto Nazionale per lo Studio e la Cura dei Tumori, Via G. Venezian 1, 20133 Milan, Italy

(Requests for offprints should be addressed to E Bajetta; email: emilio.bajetta@istitutotumori.mi.it)

\begin{abstract}
Osteoprotegerin (OPG) is a potent antiresorptive molecule that binds NF-kappaB ligand, the final effector for osteoclastogenesis. OPG production is regulated by a number of cytokines and hormones. Osteopontin (OPN) is a secreted adhesive glycoprotein involved in tumour angiogenesis, and also a non-collagenous protein involved in bone turnover. OPN serum value is associated with tumour burden and survival in advanced breast cancer patients. The short-term effects of anastrozole on OPG and OPN serum values, and the usefulness of these analytes during follow-up were studied in 34 consecutive advanced breast cancer patients receiving anastrozole $1 \mathrm{mg} /$ day. Blood samples were taken before treatment and at 2, 4, 8 and 12 weeks. OPG and OPN values were measured by ELISA. The results were analysed for all patients, and also separately for patients with (group A, 22 patients) and without (group B, 12 patients) bone metastasis. Whether the survival of all patients was related to their OPN serum values was also tested by placing patients into three groups (terciles) according to their baseline OPN values. No significant changes in OPG and OPN values were observed in the complete patient group. There was no difference in baseline OPG and OPN serum values between patients in groups A and B. In group A, a significant percentage increase in both OPG and OPN values from baseline was detected during treatment. No significant changes were reported for group $B$ patients. Furthermore, in group $\mathrm{A}$, a significant increase in both analytes was evident only for patients with progressive disease (PD). The Kaplan-Meier adjusted survival estimates for patients grouped according to tercile OPN values differed significantly $(P=0.001$, log rank test). In conclusion, in the short term, anastrozole does not seem to affect OPG and OPN serum values in patients without bone disease. OPG and OPN appear to be useful predictors of the outcome of skeletal disease and elevated OPN values may be associated with short survival in advanced breast cancer patients.
\end{abstract}

Endocrine-Related Cancer (2004) 11 771-779

\section{Introduction}

The use of third-generation aromatase inhibitors for treating postmenopausal women with oestrogen-dependent advanced breast cancer is increasing (Hamilton \& Piccart 1999). Among the available aromatase inhibitors, anastrozole plays a well-established role in clinical practice for both first- and second-line treatment of advanced disease (Buzdar et al. 2000, Nabholtz et al. 2000).
The major pharmacological effect of anastrozole is the suppression of serum oestrogen values (Plourde et al. 1994). Oestrogen suppression is responsible for the clinical efficacy of aromatase inhibitors in oestrogen-dependent tumours, although no correlation has been demonstrated between the degree of tissue and serum oestrogen suppression and clinical response (Bajetta et al. 2000, De Jong et al. 2003, Santen et al. 2003, Lønning 2004). Furthermore, it has been demonstrated that steroid 
hormones in women play a key role in regulating the equilibrium between bone formation and bone resorption (Jilka et al. 1992, Marcus 1998), and that reductions in circulating oestrogen values can induce frailness or loss of bone, as physiologically demonstrated in healthy postmenopausal women (Riggs et al. 2002). However, few data are currently available on the effects of aromatase inhibitors on bone metabolism (Bajetta et al. 2002, Heshmati et al. 2002, Eastell \& Adams 2003, Ferrari et al. 2003, Martinetti et al. 2003).

Recent studies in vitro have shown that oestradiol increases the production of the novel peptide osteoprotegerin (OPG) in human osteoblast-like cells (Hofbauer et al. 1999). Osteopontin (OPN), one of the more abundant non-collagenous proteins in bone, may also be important in the signalling pathway for the action of oestrogen on bone. It is localized to cell-matrix and matrix-matrix interfaces in mineralized tissue, where it is deposited as the result of osteoclast action, and may protect the exposed bone surface or prime it for subsequent cell-matrix interaction (Dodds et al. 1995).

OPG is a potent antiresorptive molecule that acts as a decoy receptor by blocking the interaction of RANKL (receptor activator of NF-kappaB ligand) with its functional receptor RANK, thereby inhibiting osteoclastogenesis (Anderson et al. 1997). In vitro studies have demonstrated the synthesis of OPG by stromal cell line and osteoblasts and its stimulation by oestrogen (Hofbauer et al. 1999, Saika et al. 2001).

OPN is a calcium-binding phosphoprotein which is believed to play a role in several different and apparently distinct cellular processes. Its regulation is complex at both the cellular and molecular levels, and various hormones and growth factors have been shown to influence its production (Sodek et al. 2000). Recently, expression of OPN has been linked to tumourigenesis and metastasis in several experimental animal models and human patient studies (Oates et al. 1997, Furger et al. 2001, Tuck \& Chambers 2001, Rudland et al. 2002). OPN is also known to be an important component of cell adhesion interaction, possibly mediated by the highly conserved glycine-arginine-glycine-aspartic acid-serine (GRGDS) amino-acid motif found on a number of proteins that play a role in cell adhesion (Sodek et al. 2000). Moreover, OPN appears to be an important component in the communication between osteoclasts and osteoblasts, and there is strong evidence for the involvement of OPN in the formation, migration and attachment of osteoclasts and in their resorptive activity.

In this paper, the variations of OPG and OPN circulating values during anastrozole treatment were analysed in advanced breast cancer patients, with and without bone metastasis. This study extended the biological observations previously done on the same set of patients (Bajetta et al. 2002). Furthermore, whether the survival of all patients was related to OPN serum value was tested at baseline.

\section{Patients and methods}

\section{Patients}

Between January 1998 and May 1999, 34 consecutive postmenopausal patients with advanced breast cancer (histopathologically assessed) entered the study. All patients received anastrozole (Arimidex, AstraZeneca, Macclesfield, UK) $1 \mathrm{mg}$ once daily. Postmenopausal status was defined by one of the following criteria: (1) no spontaneous menses for at least 5 years; (2) spontaneous amenorrhoea lasting at least 12 months with gonadotrophin values within the postmenopausal range (20$143 \mathrm{mIU} / \mathrm{ml}$ for follicle-stimulating hormone and 16$71 \mathrm{mIU} / \mathrm{ml}$ for luteinizing hormone); or (3) secondary amenorrhoea due to surgical treatment or radiotherapy.

Patients were required to have oestrogen receptor (ER)- and/or progesterone receptor (PR)-positive tumours. Patients with tumours of unknown receptor status could be included in the trial if they had achieved an objective response after previous hormonal treatment, with a disease-free interval of 2 years. Previous chemotherapy and/or hormonal therapy for advanced/metastatic disease were permitted. Before entering the study, the patients had to have progressive disease (PD) and any previous treatment had to have been withdrawn 3 weeks earlier. In addition, patients had to have either one bidimensionally measurable lesion or one evaluable bone lesion, and a performance status of $\leq 2$ according to the World Health Organization (WHO) score.

Patients were excluded if they had received prior treatment with any aromatase inhibitor. Concomitant treatment with corticosteroids or bisphosphonates was not allowed. Patients were also ineligible if they suffered from endocrine disorders and/or impaired liver or kidney functions.

Staging and tumour response were assessed by means of physical examination, bone scan, chest and skeletal radiographs, liver echography or computed tomography, whole blood cell count and blood chemistry. These examinations were performed at the beginning of the study and every 12 weeks thereafter. Signs, symptoms and toxicity were evaluated according to the National Cancer Institute Common Toxicity Criteria (NCI-CTC), and clinical response was evaluated in accordance with the International Union Against Cancer (UICC) criteria. Hormone-based therapy has a mainly cytostatic effect; hence, stable disease lasting over 24 weeks was also considered. 
Given that this study focuses on the biological effects of anastrozole in the short term, the clinical response considered was that obtained at 24 weeks from the beginning of the study. All patients gave their written informed consent before study enrolment. The trial was approved by the local bioethical committee and was conducted according to good clinical practice (GCP) requirements. In order to assess overall survival, patients were monitored from completion of the clinical trial until death.

\section{Biological samples, timing and specimen collection}

Peripheral blood samples were collected before starting therapy (baseline) and at 2, 4, 8, and 12 weeks during treatment. Blood withdrawals were made between 0900 and $1000 \mathrm{~h}$ after a mandatory $12 \mathrm{~h}$ fasting. Serum separation was achieved by centrifugation of the blood sample (room temperature, 2500 relative centrifugal force [rcf] for $15 \mathrm{~min}$ ). Finally, supernatants were divided into aliquots and stored at $-60{ }^{\circ} \mathrm{C}$ until the day of the assay. All assays were performed by the Laboratory of Nuclear Medicine Unit (Istituto Nazionale per lo Studio e la Cura dei Tumori, Milan, Italy).

The assay kits were assembled according to the manufacturer's guidelines. Each assay was validated in a recovery test by spiking and dilution. The accuracy of these assays was tested against serum samples with known concentrations of the tested analytes. The assays were performed in duplicate, and all samples from the same patients were analysed in the same assay batch. The assays had intra-assay coefficients of variation $<5 \%$ and interassay coefficients of variation $<6 \%$.

\section{OPG and OPN assays}

To measure OPG serum values, the OPG enzyme-linked immunosorbent assay (ELISA), produced by DRG Instruments GmbH (Harburg, Germany), and purchased from TEMA ricerca s.r.l. (Bologna, Italy), was employed. This assay is a sandwich-type ELISA for the direct determination of OPG in serum. In this assay, two highly specific antibodies against OPG (homodimeric and monomeric forms) are used. The binding antibody is attached to the wells of the microplate, and the detection antibody is labelled with biotin.

To measure OPN serum values, the enzyme immunometric assay kit produced by Assay Designs (Ann Arbour, MI, USA), purchased from TEMA ricerca s.r.1., was employed. This assay kit uses a polyclonal antibody to OPN immobilized on a microtitre plate to bind OPN in the sample. Further detection is done with a second monoclonal antibody labelled with horseradish peroxidase (HRP).

\section{Statistics}

This was a prospective, non-comparative trial, and only a descriptive analysis was performed; quantitative data are reported as median (range). The U-paired test was used to compare the values of each analyte at baseline with those during treatment $(2,4,8$, and 12 weeks). Correlation analysis among continuous variables was performed, quantifying the strength of the associations by calculating Pearson's correlation coefficient $(r)$ and the non-parametric Spearman's rank correlation coefficient (rho). In all statistical tests performed, a 5\% value of significance was used. The software SPSS for Windows, Release 6.0, by SPSS Inc (Chicago, IL, USA) was used for data management and to perform statistics. The Kaplan-Meier adjusted survival estimates were computed with the SAS statistics package (SAS Institute, Cary, NC, USA).

\section{Results}

Thirty-four patients were enrolled and deemed suitable for evaluation; their characteristics are shown in Table 1. With regard to OPG and OPN serum values, the results obtained in all patients, and also separately for patients with bone metastasis ( 22 patients, group A) and without bone metastasis (12 patients, group B), are reported. A correlation analysis was also done among OPG, OPN and the previously published results on oestrogen and bone metabolism markers (Bajetta et al. 2002). Furthermore, whether the survival of all patients was related to the OPN serum value at baseline was tested.

Taking into account all patients, no significant changes in OPG and OPN values were observed during the 12 weeks of blood sampling. Table 2 shows the median OPG and OPN serum values for all patients. There was no difference in baseline OPG and OPN serum values between patients in groups A and B. Group A showed a significant increase in both analytes during treatment, detected as percentage of variation from baseline. No significant changes were reported for group B (Fig. 1).

Figure 2 shows OPG and OPN variations versus baseline for group A according to clinical response assessed for bone metastasis, and for group B independently of the clinical response. For patients with bone metastasis (group A), five with complete (CR) or partial remissions (PR) of disease, 10 with stable disease (NC) and seven with PD were observed. Due to the low number of group B patients, the OPG and OPN variations during treatment were analysed according to clinical response, for patients with bone metastasis (group A) only. 
Table 1 Patient characteristics.

\begin{tabular}{lc}
\hline Parameter & Number of patients or value \\
\hline Median age (range) & 63 years (41-80) \\
Oestrogen receptor status: & 31 \\
Positive & 0 \\
Negative & 3 \\
Unknown & \\
Progesterone receptor status: & 20 \\
Positive & 12 \\
Negative & 2 \\
Unknown & \\
Disease site: & 19 \\
Soft tissue & 15 \\
Viscera & 22 \\
Bone & \\
Number of metastatic sites: & 16 \\
1 & 18 \\
$\geq 2$ & \\
Adjuvant treatment: & 10 \\
Chemotherapy & 17 \\
Tamoxifen & \\
Advanced treatment: & 8 \\
Chemotherapy & 7 \\
Tamoxifen & 4 \\
Chemotherapy plus tamoxifen & \\
None & \\
More than two therapies & \\
\hline Patients may be & \\
\hline
\end{tabular}

Patients may be classified in more than one group.

Table 3 shows the correlations between OPG, OPN, oestrogens (oestradiol $\left(\mathrm{E}_{2}\right)$, oestrone $\left(\mathrm{E}_{1}\right)$ and oestrone sulphate $\left.\left(\mathrm{E}_{1}-\mathrm{S}\right)\right)$, and bone metabolism markers (osteocalcin (BGP), bone alkaline phosphatase (BAP), C-terminal peptide of type I collagen (ICTP) and crosslinked N-telopeptide of type I collagen (NTx)) at baseline, considering all patients and in patients with (group A) and without (group B) bone metastasis. At baseline, no correlations between OPG, OPN and oestrogens were observed in any patient, while a weak correlation was found between OPN, ICTP and NTx, and this was maintained during treatment. The same figures were found in patients from group A with a further weak correlation between OPN and BAP. Also in group A, the correlations found at baseline were maintained during treatment. In patients from group B, a relationship were found between OPG and BGP and between OPN and $\mathrm{E}_{1}-\mathrm{S}$ at baseline only. In the same group, we found a correlation between OPN and BAP only during treatment.

Figure 3 shows that the Kaplan-Meier adjusted survival estimates of patients grouped according to tercile OPN values (lower, middle and upper tercile with OPN of $\leq 1,1.1-2.5$ and $>2.5 \mu \mathrm{g} / \mathrm{ml}$ respectively) differed significantly $(P=0.01, \log$ rank test $)$. In this cohort of 34 patients, there was a minimum follow-up period of 48 months. According to the Kaplan-Meier adjusted survival curves, the mean survivals were $34,36.5$ and 18.6 months for patients with OPN values in the lower, middle and upper terciles respectively. Mean survival time was underestimated because estimation was restricted to the largest event time. In the lower tercile group $(n=10)$ and in the middle tercile group $(n=12)$, four patients survived until 60 months, while in the upper tercile group $(n=12)$, one patient survived to 51 months (Fig. 3). Thus, the survival curves based on the tercile OPN categories suggest that elevated OPN values are associated with decreasing survival.

At baseline, median OPN serum values were 0.76, 1.75 and 3.85 for lower, middle and upper terciles respectively (Fig. 4). During treatment, OPN values were significantly different in the upper tercile with respect to the two lower ones $(P<0.0001)$ at each time point. In the lower tercile group, where $60 \%(6 / 10)$ of patients had bone metastasis, it is worth noting that one patient with soft tissue metastasis had a baseline OPN serum of $0.92 \mu \mathrm{g} / \mathrm{ml}$ that increased during treatment to $2.08,4.39,3.03$ and $3.18 \mu \mathrm{g} / \mathrm{ml}$ at $2,4,8$ and 12 weeks respectively. After 24 weeks of treatment, this patient developed bone lesions. In the middle and upper tercile groups, 58\% (7/12) and 75\% (9/12) of patients had bone metastasis respectively. Moreover, in the upper tercile group, one patient with soft tissue metastasis had a complete response, but developed bone lesions after 9 months of treatment; OPN serum values were 2.59 at baseline, and 4.32, 5.00, 2.89 and $2.74 \mu \mathrm{g} / \mathrm{ml}$ at $2,4,8$ and 12 weeks respectively. In the same group, one patient without bone lesions had a partial response to soft tissue metastasis; baseline OPN serum values were $3.68 \mu \mathrm{g} / \mathrm{ml}$ and

Table 2 Median (range) osteoprotegerin (OPG) and osteopontin (OPN) serum values in advanced breast cancer (ABC) patients treated with anastrozole.

\begin{tabular}{lccccc}
\hline & Baseline & 2 weeks & 4 weeks & 8 weeks & 12 weeks \\
\hline OPG & 4.32 & 4.43 & 4.65 & 4.38 & 4.24 \\
$(\mathrm{pmol} / \mathrm{l})$ & $(2.65-9.12)$ & $(3.11-12.55)$ & $(2.76-16.84)$ & $(2.46-13.17)$ & $(2.46-18.14)$ \\
OPNG & 1.84 & 2.01 & 1.51 & 2.14 & 1.61 \\
$(\mu \mathrm{g} / \mathrm{l})$ & $(0.54-11.34)$ & $(0.48-13.20)$ & $(0.48-12.02)$ & $(0.44-13.20)$ & $(0.44-13.20)$ \\
\hline
\end{tabular}




\section{Osteoprotegerin}

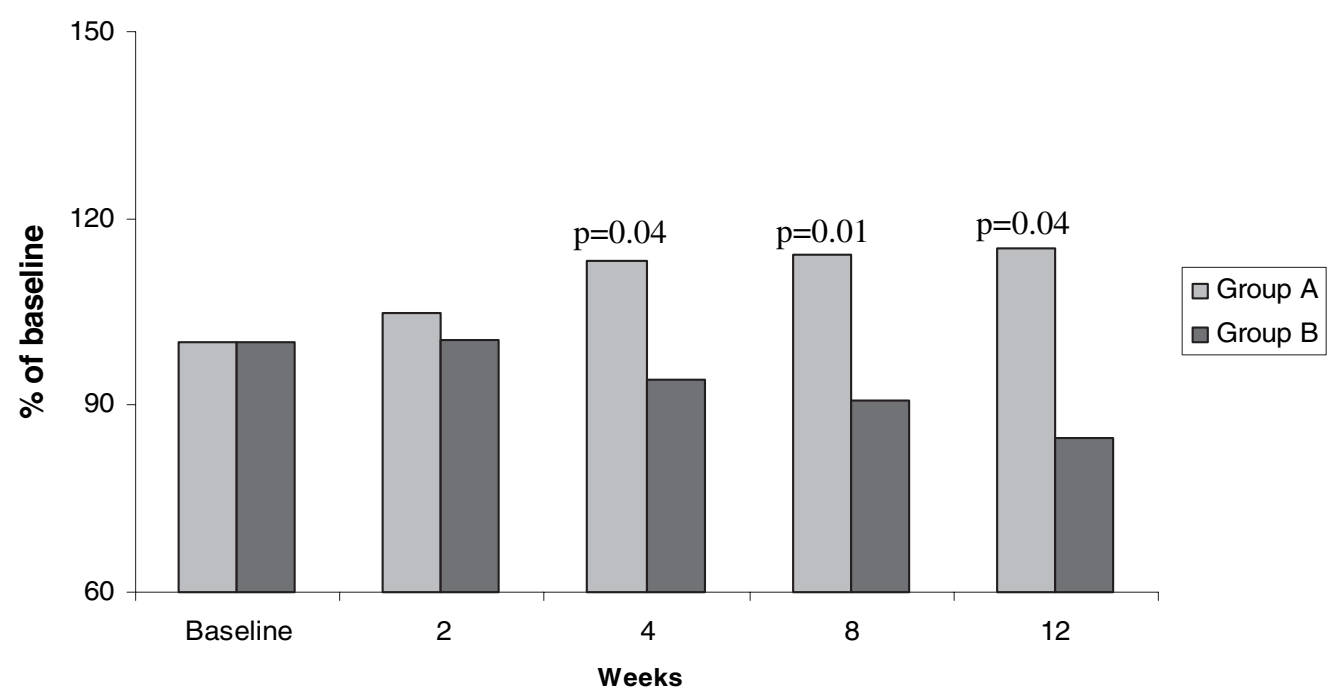

Osteopontin

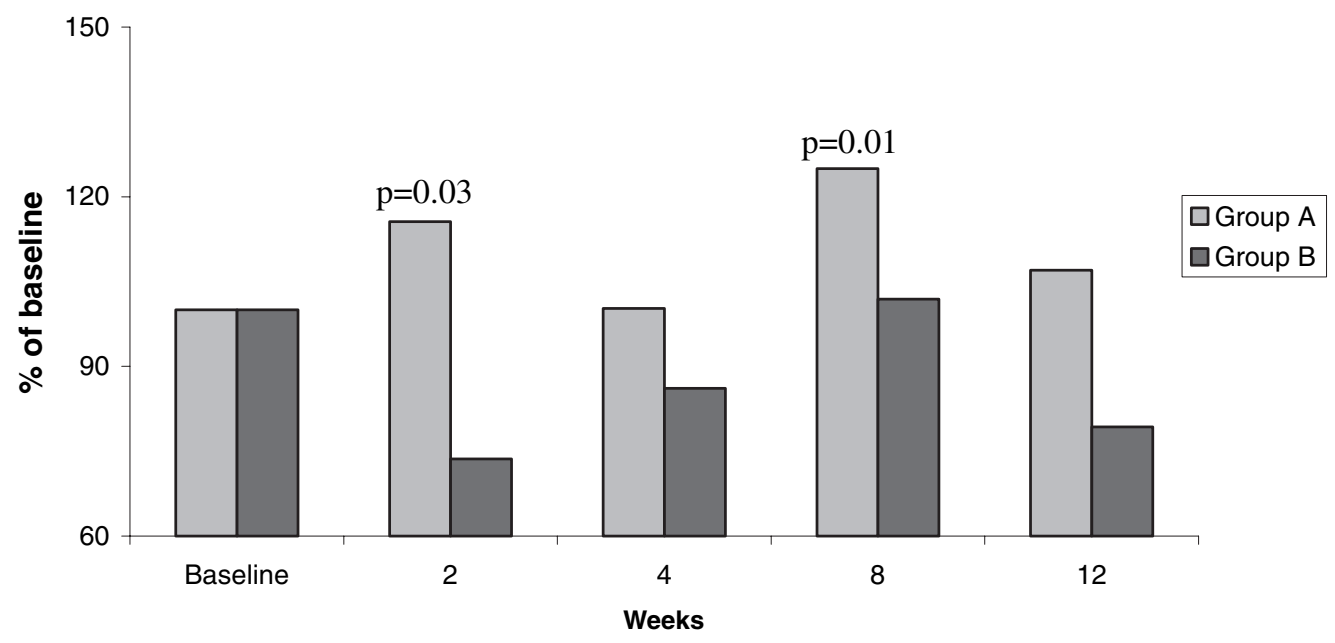

Figure 1 Osteoprotegerin (OPG) and osteopontin (OPN) median percentage variation from baseline in patients with (group A) and without (group B) bone metastasis.

decreased to $2.16,1.47,1.23$ and 2.48 at $2,4,8$ and 12 weeks respectively.

\section{Discussion}

In this study, the circulating values of two proteins (OPG and $\mathrm{OPN}$ ) involved in bone remodelling were analysed in breast cancer patients treated with anastrozole. The major pharmacological activity of anastrozole is the suppression of serum oestrogen values. In our study, anastrozole treatment significantly suppressed serum $E_{1}, E_{2}$ and $E_{1}-S$ values (Bajetta et al. 2002) after 2 weeks of treatment $(P=0.0001)$. Oestrone values decreased on average by $56.5 \%$ (range $23.1-88.8 \%$ ), $\mathrm{E}_{2}$ values by $62.6 \%$ (range $31.4-89.6 \%$ ) and $\mathrm{E}_{1}-\mathrm{S}$ values by $75.8 \%$ (range 52.4 $87.2 \%$ ). No correlation was observed between oestrogen suppression and clinical response. Significant differences in baseline serum $E_{1}, E_{2}$ and $E_{1}-S$ values were not observed 


\section{Osteoprotegerin}

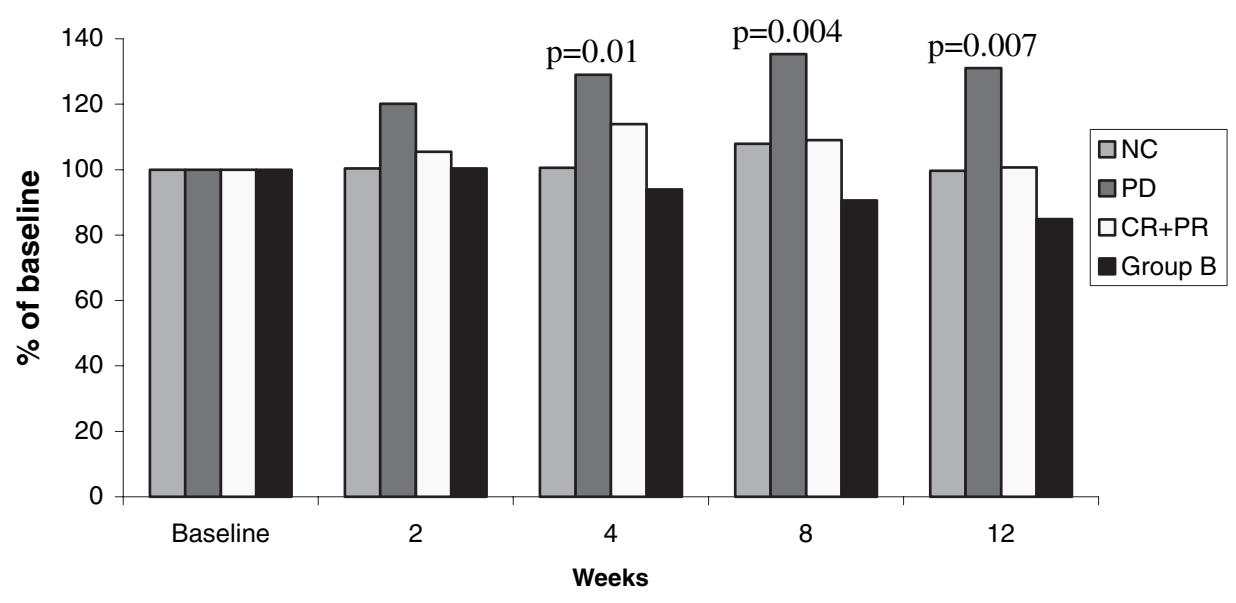

\section{Osteopontin}

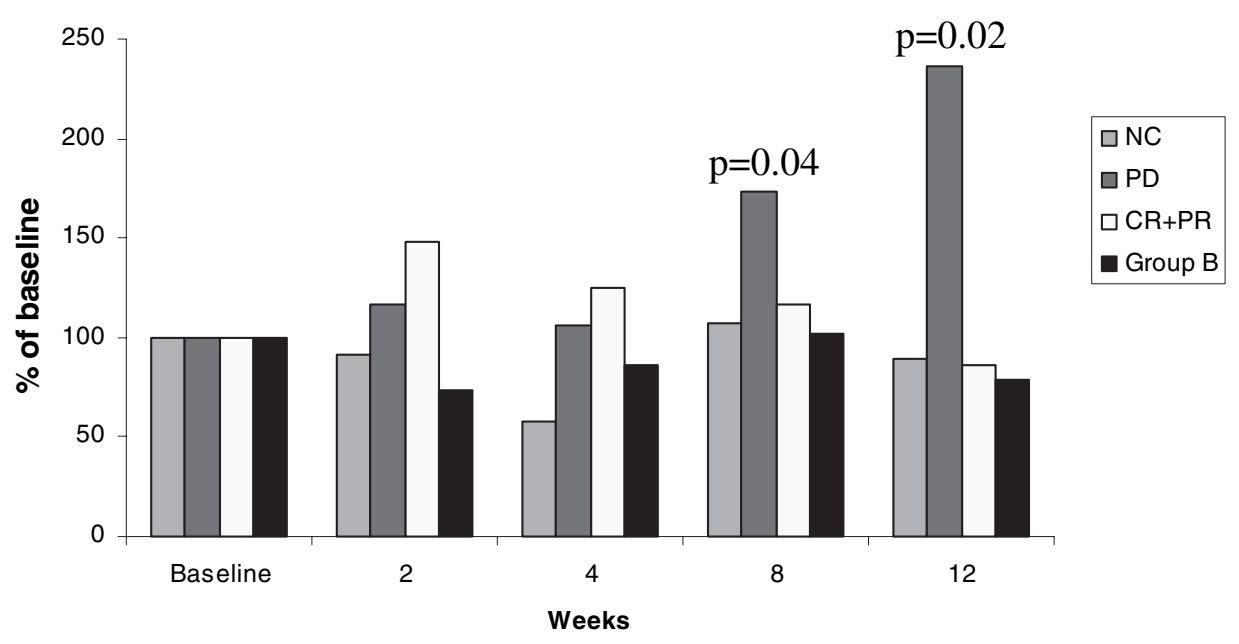

Figure 2 Osteprotegerin (OPG) and osteopontin (OPN) median percentage variation from baseline in group A, according to clinical response assessed in bone (NC, stable disease; PD, progression of disease; CR, complete remission of disease; PR, partial remission of disease) and in group $B$ patients independently of clinical response.

for patients with bone metastasis versus patients without bone metastasis, and oestrogen suppression was similar in each patient. It is well established that oestrogens have a multifunctional role influencing the growth, differentiation and function of many tissues. In women, steroid hormones play a key role in regulating the equilibrium between osteoblastic and osteoclastic activity. Oestrogens are important factors in the maintenance of bone health, as demonstrated by the observation that their deficiency at the time of menopause is associated with bone loss (Nordin et al. 1992, Riggs et al. 2002).
In patients with advanced breast cancer, the occurrence of bone metastasis uncouples bone metabolism, in either a resorptive (osteolytic) or formative (osteoblastic) way, depending on the type of disease. Metastatic bone destruction is initiated by the activation of local osteoclasts, which are themselves induced by various locally and systemically acting oncogenic mediators. Assessment of bone turnover using a panel of specific markers can help to detect bone turnover changes in tumour patients, monitor the effect of therapy, and study bone metastasis in patients affected by various neoplasms (Seregni et al. 2001). 
Table 3 Spearman's correlation coefficients (rho) and the corresponding $P$ value, among baseline circulating values of OPG, OPN, oestrogens and bone metabolism markers in all patients and separately for patients with (group A) and without bone metastasis (group B).

\begin{tabular}{|c|c|c|c|c|c|c|c|c|c|}
\hline & & OPN & BGP & BAP & ICTP & NTx & E2 & E1 & E1-S \\
\hline \multicolumn{10}{|c|}{ All patients } \\
\hline \multirow[t]{2}{*}{ OPG } & rho & -0.154 & -0.117 & 0.051 & 0.258 & 0.097 & -0.014 & 0.243 & 0.037 \\
\hline & $P$ & 0.384 & 0.509 & 0.774 & 0.141 & 0.585 & 0.935 & 0.166 & 0.833 \\
\hline \multirow[t]{2}{*}{ OPN } & rho & & 0.024 & 0.229 & 0.394 & 0.411 & 0.026 & 0.085 & -0.390 \\
\hline & $P$ & - & 0.893 & 0.194 & $0.021^{*}$ & $0.015^{\star}$ & 0.884 & 0.631 & $0.022^{*}$ \\
\hline \multicolumn{10}{|c|}{ Group A } \\
\hline \multirow[t]{2}{*}{$\mathrm{OPG}$} & rho & 0.005 & 0.032 & 0.250 & 0.335 & 0.080 & 0.119 & 0.262 & -0.130 \\
\hline & $P$ & 0.984 & 0.887 & 0.262 & 0.126 & 0.722 & 0.599 & 0.238 & 0.564 \\
\hline \multirow[t]{2}{*}{ OPN } & rho & & -0.066 & 0.444 & 0.411 & 0.441 & 0.003 & 0.207 & -0.206 \\
\hline & $P$ & - & 0.770 & $0.038^{*}$ & $0.057^{*}$ & $0.040^{*}$ & 0.990 & 0.354 & 0.358 \\
\hline \multicolumn{10}{|c|}{ Group B } \\
\hline \multirow[t]{2}{*}{$\mathrm{OPG}$} & rho & -0.427 & -0.636 & -0.329 & 0.028 & -0.210 & -0.196 & 0.322 & 0.301 \\
\hline & $P$ & 0.167 & $0.026^{*}$ & 0.297 & 0.931 & 0.513 & 0.542 & 0.308 & 0.342 \\
\hline \multirow[t]{2}{*}{ OPN } & rho & & 0.308 & -0.217 & 0.462 & 0.392 & 0.133 & -0.175 & -0.769 \\
\hline & $P$ & - & 0.331 & 0.498 & 0.131 & 0.208 & 0.681 & 0.587 & $0.003^{*}$ \\
\hline
\end{tabular}

E2, 17ß-oestradiol; E1, oestrone; E1-S, oestrone-sulphate; BGP, osteocalcin; BAP, bone alkaline phosphatase; ICTP, carboxyterminal peptide of type 1 collagen; NTx, cross-linked N-telopeptide of type 1 collagen

*Statistically significant correlations.

In this study, OPG and OPN serum values during treatment appear to relate to the presence of bone metastasis rather than to anastrozole treatment. Additionally, a significant increase in OPG and OPN serum values was noted only in patients with bone metastasis (group A). Increases in OPG appear to relate to the clinical response. During the observation period, only patients with PD showed a significant OPG increase. No significant modification was noted for the other patients. A possible explanation of this observation lies in the

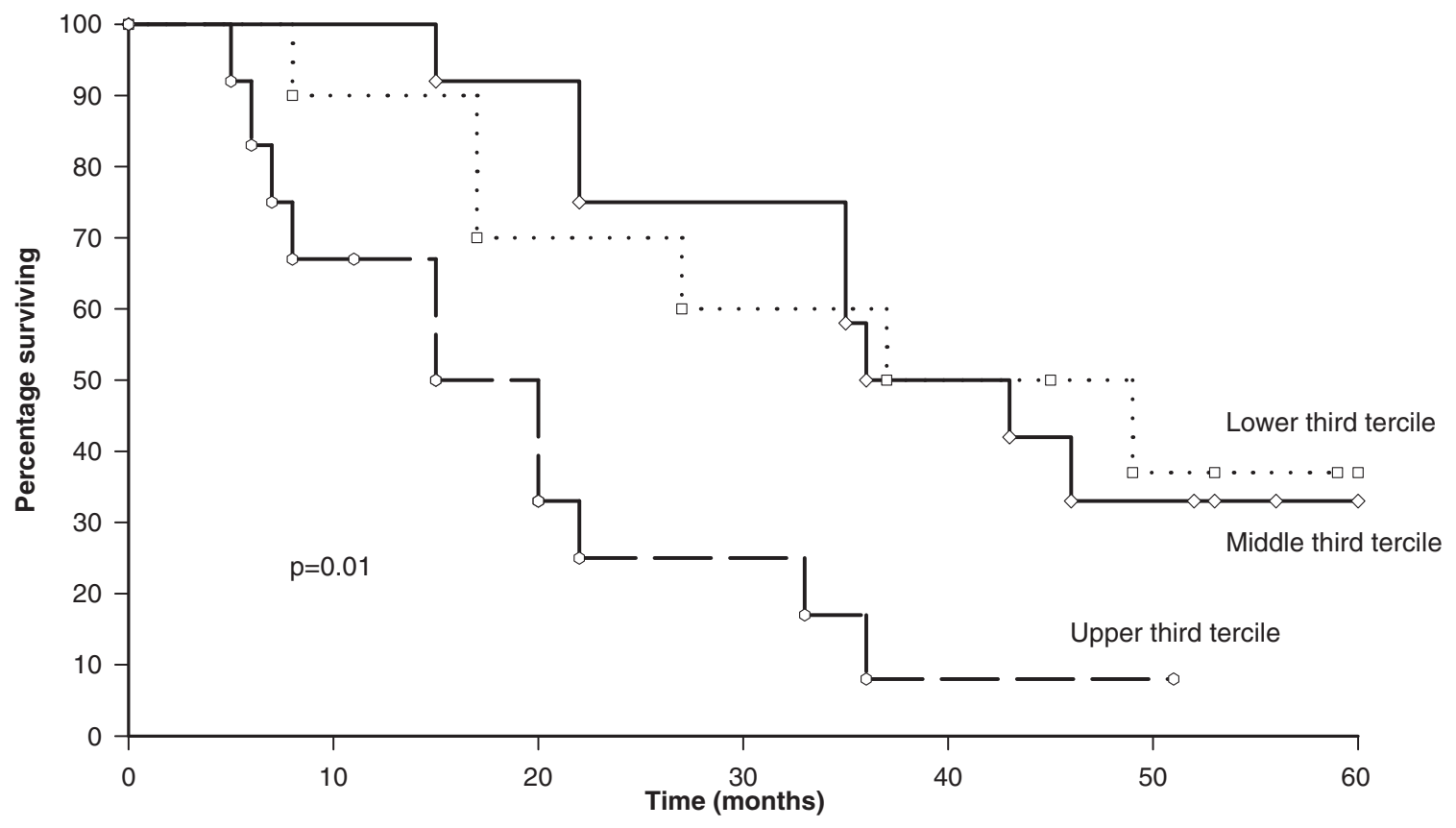

Figure 3 Kaplan-Meier adjusted percentage survival as function of time (months) of patients grouped into tercile serum osteopontin (OPN) baseline values. 


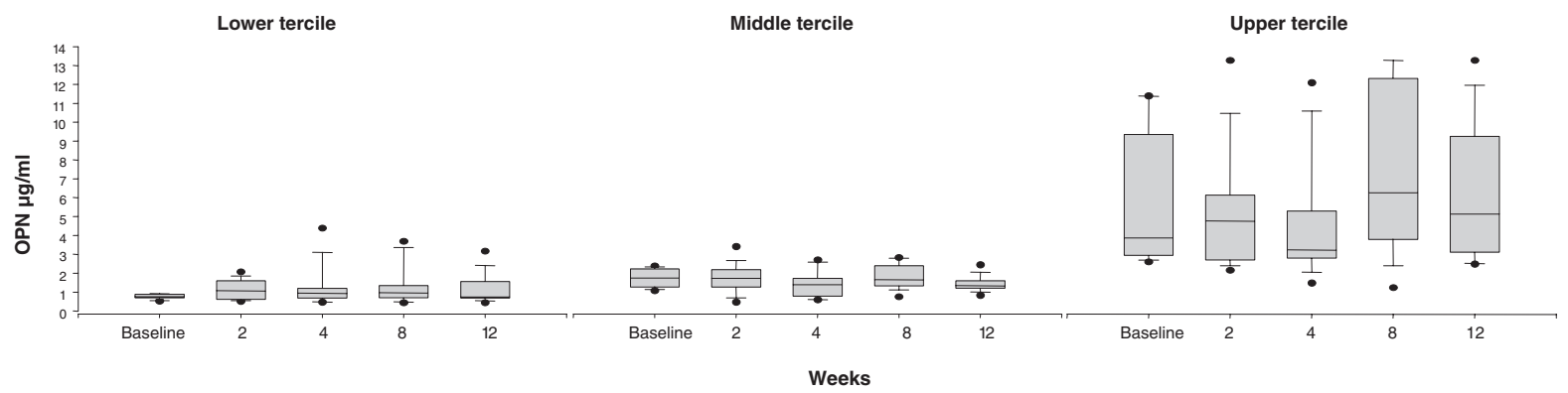

Figure 4 Osteopontin (OPN) serum value behaviour in patients grouped according to tercile OPN baseline values. The box plots describe the OPN distribution in the study population; the line into the box is the median value of OPN. The box ends represent the 25th and 75th percentiles, and the lines the 5th and 95th percentiles respectively. The points represent the outliers.

attempt to balance increased osteoclastic activity, as evidenced by an increase in serum concentration of bone resorption markers (Bajetta et al. 2002). A similar scenario has been hypothesized by other authors for osteoporotic women (Yano et al. 1999, Arrighi et al. 2000). OPN serum value behaviour was similar to OPG, and this analyte showed a significant increase only in patients with PD.

At baseline, the bone resorption markers ICTP and NTx showed a weak but significant relationship with OPN both in all the patients and in patients with bone metastasis (group A). It is worth noting that in group A patients these correlations were maintained also during treatment. Furthermore, in group A patients, we observed a positive significant correlation of BAP with OPN at all examination times. These findings seem to suggest that in subjects with bone metastasis an activation of osteolytic and osteoblastic processes occurs. By contrast, in group B patients, both at baseline and during treatment, the lack of a relationship seems to suggest an activation of bone remodelling.

In order to determine whether the value of OPN present in blood might also be of predictive value, patients were grouped into upper, middle and lower thirds of OPN baseline values. There was a significant association between elevated serum OPN values and shorter survival $(P=0.01)$ with a follow-up of 60 months. These data agree with those of Singhal et al. (1997), who reported similar findings in 70 early breast cancer patients with a follow-up of 20 months.

In conclusion, our results suggest that in the short term OPG and OPN are not modified by the high degree of oestrogen suppression induced by anastrozole treatment, but may be related to clinical outcome. OPN may also be associated with shorter survival. Our results suggest the need for a large prospective trial to address the utility of measuring OPG and OPN serum values in breast cancer patients.

\section{Acknowledgements}

The authors would like to thank the Italian Trials in Medical Oncology (ITMO) Scientific Service for their editorial assistance, Mrs B. Formisano for her assistance in data managing, and Dr R. Artioli (AstraZeneca Italy), who partially supported this research.

\section{References}

Anderson DM, Maraskovsky E, Billingsley WL, Dougall W, Tometsko ME, Roux ER, Teepe MC, DuBose RF, Cosman D \& Galibert L 1997 A homologue of the TNF receptor and its ligand enhance T-cell growth and dendritic-cell function. Nature 390 175-179.

Arrighi H, Khosla S, Melton LJ, Riggs BL, Bekker PJ \& Dunstan CR 2000 The relationship between circulating osteoprotegerin and estrogen in postmenopausal women. Journal of Bone and Mineral Research 15 S441.

Bajetta E, Zilembo N, Bichisao E, Martinetti A, Buzzoni R, Pozzi P, Bidoli P, Ferrari L \& Celio L 2000 Tumor response and estrogen suppression in breast cancer patients treated with aromatase inhibitors. Annals of Oncology 11 1017-1022.

Bajetta E, Martinetti A, Zilembo N, Pozzi P, La Torre I, Ferrari L, Seregni E, Longarini R, Salvucci G \& Bombardieri E 2002 Biological activity of anastrozole in postmenopausal patients with advanced breast cancer: effects on estrogens and bone metabolism. Annals of Oncology 13 1059-1066.

Buzdar A, Nabholtz J, Robertson J, Thürlimann B, Bonneterre J, von Euler M, Steinberg M \& Webster A 2000 Anastrozole (Arimide ${ }^{\mathrm{TM}}$ ) versus tamoxifen as first-line therapy for advanced breast cancer (ABC) in post-menopausal (pm) women - combined analysis from two identically designed multicenter trials. Proceedings of the American Society of Clinical Oncology 19 154a (Abstract 609D).

De Jong PC, Blankenstein MA, Nortier JWR, Slee PHThJ, van de Ven J, van Gorp JMHH, Elbers JRJ, Schipper MEI, Blijham GH, Thijssen JHH, Lu Q, Jelovac D \& Brodie AM 2003 The relationship between aromatase in primary breast tumors and response to treatment with aromatase inhibitors 
in advanced disease. Journal of Steroid Biochemistry and Molecular Biology 87 149-155.

Dodds RA, Connor JR, James IE, Rykaczewski EL, Appelbaum E, Dul E \& Gowen M 1995 Human osteoclasts, not osteoblasts, deposit osteopontin onto resorption surfaces: an in vitro and ex vivo study of remodeling bone. Journal of Bone and Mineral Research 10 1666-1680.

Eastell R \& Adams J 2003 Bone mineral density and bone marker subprotocol. In History and Advancement of Anastrozole in the Treatment of Breast Cancer, Ch 11. Eds A Buzdar \& M Baum. London: Royal Society of Medicine.

Ferrari L, Bajetta E, Martinetti A, Celio L, Longarini R, La Torre I, Buzzoni R, Gattinoni L, Seregni E \& Bombardieri E 2003 Could exemestane affect insulin-like growth factors, interleukin 6 and bone metabolism in postmenopausal advanced breast cancer patients after failure of aminogluthimide, anastrozole or letrozole? International Journal of Oncology 22 1081-1089.

Furger AK, Menon RK, Tuck AB, Bramwell VHC \& Chambers AF 2001 The functional and clinical roles of osteopontin in cancer and metastasis. Current Molecular Medicine 1 621-632.

Hamilton A \& Piccart M 1999 The third-generation non-steroidal aromatase inhibitors: a review of their clinical benefits in the second-line hormonal treatment of advanced breast cancer. Annals of Oncology 10 377-384.

Heshmati HM, Khosla S, Robins SP, O'Fallon WM, Melton LJ III \& Riggs BL 2002 Role of low levels of endogenous estrogen in regulation of bone resorption in late postmenopausal women. Journal of Bone and Mineral Research 17 172-178.

Hofbauer LC, Khosla S, Dunstan CR, Lacey DL, Spelsberg TC \& Riggs BL 1999 Estrogen stimulates gene expression and protein production of osteoprotegerin in human osteoblastic cells. Endocrinology 140 4367-4370.

Jilka RL, Hangoc G, Girasole G, Passeri G, Williams DC, Abrams JS, Boyce B, Broxmeyer H \& Manolagas SC 1992 Increased osteoclast development after estrogen loss: mediation by interleukin-6. Science 257 88-91.

Lønning PE 2004 Aromatase inhibitors in breast cancer. Endocrine-Related Cancer 11 179-189.

Marcus R 1998 New perspectives on the skeletal role of estrogen. Journal of Clinical Endocrinology and Metabolism $\mathbf{8 3}$ 2236-2238

Martinetti A, Zilembo N, Ferrari L, Massimini G, Polli A, La Torre I, Giovanazzi R, Pozzi P, Bidoli P, De Candis D, Seregni E \& Bombardieri E 2003 Bone turnover markers and insulin-like growth factor component in metastatic breast cancer: results from a randomized trial of exemestane vs megestrol acetate. Anticancer Research 23 3485-3491.

Nabholtz JM, Buzdar A, Pollak M, Harwin W, Burton G, Mangalik A, Steinberg M, Webster A \& von Euler M 2000 Anastrozole is superior to tamoxifen as first-line therapy for advanced breast cancer in postmenopausal women: results of a North American multicenter randomized trial. Arimidex Study Group. Journal of Clinical Oncology 18 3758-3767.

Nordin BE, Need AG, Bridges A \& Horowitz M 1992 Relative contributions of years since menopause, age, and weight to vertebral density in postmenopausal women. Journal of Clinical Endocrinology and Metabolism 74 20-23.

Oates AJ, Barraclough R \& Rudland PS 1997 The role of osteopontin in tumorigenesis and metastasis. Invasion Metastasis 17 1-15.

Plourde PV, Dyroff M \& Dukes M 1994 Arimidex: a potent and selective fourth-generation aromatase inhibitor. Breast Cancer Research and Treatment 30 103-111.

Riggs BL, Khosla S \& Melton LJ 2002 Sex steroid and the construction and conservation of the adult skeleton. Endocrine Reviews 23 279-302.

Rudland PS, Platt-Higgings A, El-Tanani M, De Silva Rudland S, Barraclough R, Winstanley JHR, Howitt R \& West CR 2002 Prognostic significance of the metastasis-associated protein osteopontin in human breast cancer. Cancer Research $623417-3427$.

Saika M, Inoue D, Kido S \& Matsumoto T 2001 17 $\beta$-Estradiol stimulates expression of osteoprotegerin by a mouse stromal cell line, ST-2, via estrogen receptor- $\alpha$. Endocrinology 142 2205-2212.

Santen JR Song RX, Zhang Z, Kumar R, Jeng MH, Masamura S, Yue W \& Berstein L 2003 Adaptive hypersensitivity to estrogen: mechanism for superiority of aromatase inhibitors over selective estrogen receptor modulators for breast cancer treatment and prevention. Endocrine-Related Cancer 10 111-130.

Seregni E, Martinetti A, Ferrari L \& Bombardieri E 2001 Clinical utility of biochemical marker of bone remodelling in patients with bone metastases of solid tumors. Quarterly Journal of Nuclear Medicine 45 7-17.

Singhal H, Bautista DS, Tonkin KS, O'Malley FP, Tuck AB, Chambers AF \& Harris JF 1997 Elevated plasma osteopontin in metastatic breast cancer associated with increased tumor burden and decreased survival. Clinical Cancer Research 3 605-611.

Sodek J, Ganss B \& McKee MD 2000 Osteopontin. Critical Reviews in Oral Biology and Medicine 11 279-303.

Tuck AB \& Chambers AF 2001 The role of osteopontin in breast cancer: clinical and experimental studies. Journal of Mammary Gland Biology and Neoplasia 6 419-429.

Yano K, Tsuda E, Washida N Kobayashi F, Goto M, Harada A, Ikeda K, Higashio K \& Yamada Y 1999 Immunological characterization of circulating osteoprotegerin/ osteoclastogenesis inhibitory factor: increased serum concentrations in postmenopausal women with osteoporosis. Journal of Bone and Mineral Research 14 518-527. 